\title{
Targeting Molecular Pathways for Prevention of High Risk Breast Cancer: A Model for Cancer Prevention
}

\author{
Shayna Showalter and Brian J. Czerniecki \\ Department of Surgery and the Rena Rowan Breast Center, Abramson Cancer Center, \\ Perelman School of Medicine, University of Pennsylvania, Philadelphia, PA, \\ USA
}

\section{Introduction}

\subsection{Role of vaccines in disease prevention and cancer prevention}

Vaccine therapy is traditionally designed to be given prophylactically in order to prevent infectious diseases. Vaccines formulated against pathogens known to cause disease have been successfully created and implementation policies have been effective in the prevention and eradication of many life-threatening diseases. A well-know example of the success that is possible with a well constructed vaccine and an efficient vaccination strategy is the poliomyelitis vaccine. Poliomyelitis was first documented in the late 19th century and it quickly reached the level of causing annual global endemics. The vaccine was introduced in the United States in 1955 and was associated with an immediate reduction in the disease and eventual the elimination of wild-type polioviruses in the United States by 1972. Subsequent global expansion of polio vaccination has resulted in a drastic reduction of documented cases of the disease as well as eradication of wild type 2 poliovirus [1].

A natural next step after the development of prophylactic vaccines to prevent infectious disease was the emergence of vaccines used against specific infectious agents that are known to cause cancer. An estimated $12 \%$ of human cancers are attributable to viral infections [2]. Viral infections that are known to cause cancer include human papillomavirus (HPV), hepatitis B virus (HBV), hepatitis C virus (HCV), Epstein-Barr virus (EBV), and Kaposi's sarcoma-associated herpes virus (KSHV). Safe and effective vaccines have been developed against two oncovirus, HPV to prevent HPV-associated cervical carcinoma and $\mathrm{HBV}$ to prevent $\mathrm{HBV}$-associated hepatocellular carcinoma.

\subsection{Primary versus secondary prevention}

A small number of cancers are directly associated with exposure to an oncovirus. Vaccinating against these viruses and preventing infection and subsequent cancer formation is referred to as 'primary cancer prevention'. This term also encompasses the theoretic potential of vaccinating against non-infectious cancers prior to tumorigenesis. Most cancers do not have a direct link to one specific pathogen. Therefore, experimental cancer vaccines 
are designed to stimulate an immune response against pathogens from established tumors. The creation of a vaccine against an established tumor, either invasive or pre-invasive, is referred to as 'secondary cancer prevention'. The goal of secondary cancer prevention is to use active specific immunotherapy to eradicate cancer cells without causing harm to healthy tissues. Successful secondary cancer prevention can have of a number of goals including inhibiting the evolution of pre-invasive to invasive disease, impeding the progression of disease and the formation of metastases, and increasing patient survival.

\subsection{Breast cancer background and potential for vaccine therapy}

Breast cancer remains the most common non-skin cancer diagnosis and the second leading cause of cancer related death in women [3]. Major improvements in the surgical and adjuvant treatment of breast cancer during recent decades have resulted in improved disease-free and overall survival for breast cancer patients. Morbidity from surgery, chemotherapy and radiation is substantial and even with optimal current treatments approximately 40,000 women a year succumb to breast cancer [3].

A general challenge to constructing an effective cancer vaccine is that all tumor cells contain self-antigens that vary from normal tissue by mutation or expression level and therefore cancer cells are able to evade immune surveillance. It is essential to find tissue and tumor specific molecules that are capable of stimulating an immune response. Vaccination efforts are often focused on high risk cancers where the clinical impact can be the greatest. Immunotherapy, which involves actively manipulating the immune system to target tumors, promises the potential for a safe and effective adjuvant treatment for patients with high risk breast cancer.

\subsection{Molecular phenotypes of breast cancer}

Elucidation of the molecular basis of carcinogenesis has identified that breast cancer and probably all solid tumors exist as discrete molecular subtypes rather than a single disease. Breast cancer is a heterogeneous disease and several microarray profiling studies have identified distinct subtypes of breast tumors that are associated with different clinical outcomes [4-7]. The implications of classifying tumors based on gene profiling are both therapeutic and predictive. Gene expression profiling facilitates both the prediction of patient outcome and the selection of patients that will benefit from specific adjuvant therapies.

Breast tumors are typically classified into five distinct genetic subtypes based on immunphenotype and the expression of the following receptors; estrogen (ER), progesterone (PR), human epidermal growth factor receptor-2/neu (HER-2), cytokeratin 5/6 (CK5/6) and epidermal growth factor (EGFR). Luminal A cancers are ER positive and/or PR positive, HER-2 negative and Grades 1 or 2. Luminal $B$ cancers are (a) ER positive and/or PR positive and HER-2 positive or (b) ER positive and/or PR positive and HER-2 negative and high grade. HER-2 type cancers stain negative for ER and PR and positive for HER-2. Basallike tumors have no staining for ER, PR and HER-2, but do stain positive for CK 5/6 and/or EGFR. Tumors that have no staining for all 5 markers are referred to as Unclassified [4, 8, 9]. These molecular phenotypes of breast carcinoma can be delineated with routine immunohistochemical markers. Substantial differences in the survival of patients with different subtypes have been reported. Luminal A tumors have a significantly better 5- and 
10- year survival compared to Luminal B, HER-2, Basal-like and unclassified tumors [4]. In addition, certain ductal carcinoma in situ (DCIS) lesions over express HER-2, which results in a more rapid progression to invasive disease [11] and higher risk of recurrence [10, 13].

Anti-estrogen therapy is used for primary and secondary prevention of luminal tumors, but there are currently no similar options for prevention of the high risk tumors (Luminal B, Basal, HER-2). Trastuzumab is a human epidermal HER-2-targeted monoclonal antibody that has been shown to decrease recurrence and improve survival when used in the adjuvant setting combined with chemotherapy to treat patients with invasive disease that over-express the HER-2 protein [14]. The effect of trastuzumab has been postulated to be mediated by antibody-dependent cytotoxicity (ADCC) [15]. Unfortunately, this regimen is often not curative $[14,16]$ and patients can become resistant to therapy and ultimately fail [17] . A protein in the HER family would be an ideal target for a breast cancer vaccine. The HER family of tyrosine receptor kinases of which HER-1, HER-2, HER-3 and HER-4 are members make intriguing targets as these molecules are implicated in HER-2 and Basal-type breast cancers and also play a significant role in the development of some of the Luminaltype breast cancers.

\section{Cancer and the immune response}

The immune system is a complex and overlapping cellular network that protects against foreign pathogens and closely regulates self-tolerance. The innate system represents the first line of defense to tissue injury and foreign pathogens. It is comprised of natural barriers, cytokines, neutrophils, macrophages, dendritic cells (DCs), and natural killer (NK) cells [18, 19]. The innate response also includes the activation of the complement pathway. The early, antigen-nonspecific response of the innate immune system is necessary for the activation of the adaptive immune system which is comprised of B- and T- lymphocytes that express antigen-specific receptors and are ultimately responsible for producing and maintaining immunologic memory [20].

\subsection{Cancer response to the immune system: exploitation and evasion and editing}

In order for cancer cells to survive they must be able to either evade the immune system or to exploit it in a way that causes immune cells to actually enhance tumor growth. The immune response to neoplastic development is often described as paralleling the body's response to inflammation. It can be simplistically divided into an 'acute' and 'chronic' reaction. Epithelial cancer progression and eradication, similar to an inflammatory reaction, are regulated by both the innate and adaptive immune systems [21]. The specific immune cells involved paradoxically enhance and eliminate carcinogenesis. Accumulated data from animal and human studies has shown that the acute immune response to tumor growth is an anti-neoplastic process, comprised of CD8+ T cells, $\mathrm{T}_{\mathrm{H}} 1$ cells and NK cells [22].

Continued epithelial cancer development leads to dysregulation between the two subsets of the immune system and excessive activation results in an immune response that is similar to the body's response to chronic inflammation. Chronic activation of innate immune cells is associated with an ongoing infiltration cells that facilitate the survival of neoplastic cell survival by stimulating angiogenesis, inflammation and proliferation [19-23]. The chronic activation of the innate immune system also directly contributes to cancer development by 
suppressing the anti-tumor adaptive immune response (CD8+ T cells, $\mathrm{T}_{\mathrm{H}} 1$ cells, NK cells) and allowing tumor cells to escape from surveillance. One type of innate immune cells, myeloid suppressor cells, are known to accumulate in the peripheral blood of patients with cancer [24, 25]. Myeloid suppressor cells directly inhibit T lymphocytes and therefore inhibit the anti-tumor environment produced by innate immune cells $[25,26]$. These cells also promote tumor growth by assisting in angiogenesis [27].

In addition to suppressing the anti-tumor effects of the adaptive immune system, chronic activation of the cells of the innate immune (B cells, $\mathrm{T}_{\mathrm{H}} 2$ cells) response actually promotes tumor development [21]. Multiple population based studies have definitively linked chronic inflammatory conditions with the development of certain cancers. For example Helibacter pylori infection and gastric cancer, inflammatory bowel disease and colon cancer, and hepatitis and hepatocellular carcinoma [28-30]. Subsequent studies that revealed an inverse relationship between long-term usage of anti-inflammatory medications and a decreased cancer risk support the support the direct association between chronic inflammation and cancer development [31]. Through a variety of cellular mediators, chemokines and cytokines, innate immune cells and $\mathrm{T}_{\mathrm{H}} 2$ cells are able to create a pro-tumor microenvironment that favors cell proliferation, genomic instability and malignant conversion [32].

In addition to exploiting the immune system to stimulate tumor growth, cancer cells must also be able to evade the immune response. Prolonged activation of the innate immune cells results in subsequent suppression of the anti-tumor adaptive immune response, therefore allowing tumors cells to avoid specific immune surveillance. Neoplastic lesions attract regulatory $\mathrm{T}$ cells that suppress cytotoxic $\mathrm{T}$ cells [33]. Furthermore, cancer cells avoid immune surveillance by over expression or mutation of self-peptides. These non-foreign antigens are only weakly immunogenic and thus evade the host immune response or do not induce an immune specific response in the same way that a completely foreign antigen would.

Growing tumors are phenotypically sculpted by the immune system. One of the risks of cancer immunotherapy is that it can result in 'immunoediting', whereby the immune response sculpts cancer cells into a more aggressive phenotype [34, 35]. Surviving tumor cells acquire the ability to evade immune recognition through selective pressures that favor the survival and reproduction of cancer cells that lack the selected antigen. In considering an immunotherapy target it is important to select an antigen that is central to the survival of the tumor cell or that contributes to the aggressiveness of the disease. Therefore, when the cells adapt, only variants that do not express the antigen will survive. This creates a tumor that consists of cells that are unable to survive or that have a phenotype that is associated with a better clinical prognosis [36]. This process of immunoediting explains in part why targeting only single antigens has resulted in limited clinical success.

\subsection{General potential for vaccine therapy}

The intimate relationship between cancer and the immune system illustrates the potential for an effective immunotherapeutic agent, such as a cancer vaccine, to harness the immune system and then to manipulate the immune cells to create a strong anti-tumor environment. Traditional vaccines are designed to be prophylactic. The immunogens in the vaccines are 
administered prior to disease exposure and effective immunity is created before infection. In contrast, cancer vaccines are intended to stimulate active immunity only after tumors cells are already present and established. Oftentimes, vaccines are not given until the cancer has spread systemically. Also, unlike bacteria or other microbes for which vaccines are used, all tumor cells also express antigens that are very similar to established self-antigens. The ideal vaccine target would be an antigen that is present only on cancer cells and not on normal cells. Since this is uncommon, one of the largest challenges in vaccine development is breaking immune tolerance without inducing autoimmune reaction that would be harmful to healthy tissues. Several vaccine approaches have been established for a multitude of cancers in both early and late stages.

\section{Immune response in relation to invasive and in situ breast cancer}

Breast cancer, both the in situ and invasive forms, is an ideal target for vaccine therapy since this disease creates a significant public health burden. There is potential for vaccines to inhibit the progression of in situ disease into invasive cancer. The ultimate goal would be to prevent the formation of breast cancer altogether. Central to success of using immunotherapy to treat breast cancer is that breast tumors have already been established to be relatively immunogenic and the growing tumors are subject to immunosurveillance. Tumor antigens that are over-expressed or mutated in breast cancer cells initiate the development of a tumor-specific adaptive immune response [23, 37, 38]. T-cells that recognize these antigens have been isolated from breast cancer patients [39, 40]. As further evidence that the cell-mediated immune reaction has an important role in breast cancer development and clinical outcome, lymphocyte infiltration has been shown to be associated with improved survival in breast cancer patients [41]. Recent data by Mahmoud et al confirmed that the presence of an efficient T-cell-mediated immune response is associated with breast cancer outcomes [42]. This study, a retrospective review of immunohistochemical staining from nearly 2000 patients with invasive breast cancer who received standard surgical and adjuvant treatment revealed that a higher number of $\mathrm{CD}^{+} \mathrm{T}$ lymphocytes infiltrating the tumor of adjacent stroma was independently associated with longer survival in patients with invasive breast cancers.

In addition to being immunogenic, other aspects of breast cancer make it a good model for the development of a high-impact cancer vaccine, especially for patients with early stage disease. First, solid tumor cancer vaccines have had limited success when used to treat advanced or metastatic disease [43]. Breast cancer is most frequently treated with surgery and radiation therapy, which greatly decreases the disease burden, even in advanced cases. This tumor debulking provides a greater potential for disease eradication by competent immune cells. Second, the typical slow growing nature of most breast cancers allows for the expansion of immune cells over time with repeated vaccine boosters. Therefore, effective levels of active and immune competent cells can be achieved before the disease becomes systemic.

Although most cancer vaccines have been developed to treat metastatic and systemic disease, there are a number of theoretical benefits to instead delivering vaccine therapy to patients with limited, microscopic cancer burden (as neoadjuvant therapy) in the absence of bulky disease. For instance, immune-competent patients would be able to produce a significant response comprised of antigen-competent $\mathrm{T}$-cells that can rapidly expand when 
presented with low antigen levels of early disease or early recurrence [44]. In addition, patients with early stage breast cancer do not require aggressive adjuvant therapy. The immune response in vaccinated patients with early stage cancers will not be compromised by the limitations of these cytotoxic treatments, most importantly the long-term reductions in functioning B and T lymphocytes [45-47]. There has therefore been a shift in the field of immunotherapy towards the treatment of patients with minimal disease and the prevention breast cancer formation.

\subsection{Immunotherapy and ductal carcinoma in situ}

With the increasing use of screening mammography, DCIS, the pre-invasive form of breast cancer, has become the most frequently diagnosed breast malignancy. DCIS is a heterogeneous disease both in terms of nuclear grade and expression of cell-surface receptors. DCIS is the non-obligate precursor of invasive ductal carcinoma. Low-grade DCIS may develop into invasive cancer slowly or not all. In contrast, high-grade DCIS almost always develops into invasive disease and often requires more aggressive surgical and adjuvant therapy [48].

Currently, in most clinical practices, DCIS lesions are examined for the over-expression of ER and PR. Interest in the correlation between the molecular biology of DCIS and its clinical aggressiveness has led to staining for other markers. The additional information about potential antigen targets on DCIS is useful to guide development of novel adjuvant vaccines against DCIS. Specifically, for the past 6 years we have routinely stained all of the tumors from our DCIS patients for the over-expression of HER-2. Other biologic markers that are may have prognostic significance include HER 1-4, Ki67, p21, bcl-2, p16 and COX-2, c-myc and survivin $[10,13,49]$.

Multiple recent studies have concentrated on determining molecular phenotypes for DCIS similar to those described for invasive breast cancer. In 2010 Tamimi et al published a large case series of DCIS and invasive breast tumors that were analyzed using tissue microarray and immunostaining for ER, PR, HER-2, CK 5/6 and EGFR. The authors concluded that the same 5 molecular phenotypes used to describe invasive cancer were all identified among the cases of DCIS. The prevalence of the lesions was not consistent between the DCIS and invasive tumors; the Luminal A phenotype was significantly more frequent among the invasive cancers and the Luminal B and HER-2 molecular phenotypes were more frequent in DCIS. The triple negative (Basal-like) phenotype is very uncommon in DCIS [50]. This is consistent with other studies that show a higher prevalence of HER-2 over expression in DCIS compared to invasive breast cancer [51-53]. Additional work has expanded the traditional molecular profiling to incorporate many more biomarkers that have been found to be biologically relevant to invasive breast cancer, including p53, bcl-2 and P-cadherin. Bcl2 was found to be one of the most common genes to be up regulated in the welldifferentiated sample of DCIS [53] and has also been reported to be a predictor of good prognosis in invasive breast cancers [54].

DCIS is a particularly attractive vaccine target because the elimination of this disease prevents the subsequent development of invasive breast cancer. In addition, a novel neoadjuvant vaccine would be ideal to reduce size of these lesions prior to surgery. This could theoretically decrease the amount of breast tissue required to obtain clear margins 
during surgical excision and could also prevent the risk of subsequent recurrence. In general, DCIS patients are often otherwise healthy and are therefore able to mount an immune response to vaccination. The long latency period between the diagnosis of in situ disease and the development of invasive breast cancer, as well as the minimal disease burden of DCIS, provides an ideal therapeutic window to administer preventative vaccines. This strategy of treating early disease is applicable to early invasive breast cancer as well as to other cancers with indolent courses or those that are diagnosed in early stages, such as prostate and colon cancers, chronic leukemia and lymphomas [48, 55].

\section{Selection of a tumor antigen target}

The selection of an appropriate target tumor antigen is paramount to the success of any cancer vaccine. The ideal vaccine would stimulate a significant immune response without causing autoimmunity and without a detrimental side effect profile. One strategy to avoid autoimmunity and to enhance the specificity of the vaccine is to target tumor antigens that are overexpressed in cancer cells but have minimal expression in normal cells. As mentioned in a previous section, one challenge to targeting oncogenic molecules is that these tumor associated antigens are usually only weakly immunogenic and are therefore capable of evading the immune response, or the immune system can immunoedit the tumors leaving behind antigen negative tumor cells. Many of the breast cancer tumor antigens are also overexpressed in other cancers of epithelial cell origin (colon cancer and ovarian cancer). Previously targeted peptides in the experimental production of vaccines against breast cancer include Mucin (MUC)-1, Her-2, carcinoembryonic antigen (CEA), and survivin [56-58].

\subsection{The EGF receptor family}

The epidermal growth factor receptor (EGFR) family is a group of four related transmembrane receptor tyrosine kinases that have been implicated in the development of a multiple solid malignancies and have subsequently been targeted in a variety of novel therapeutics [59]. The EGFR family consists of HER-1 (also known as ERBB1), HER-2 (ERBB2), HER-3 (ERBB3) and HER-4 (ERBB4). These receptors bind 13 different ligands and form a number of different dimers between the family members. Ligand binding and dimerazation initiates various intracellular signaling pathways that affect numerous cellular processes involved in cell survival and proliferation. The oncogenic effects of the EGFR proteins result from amplification, over expression or mutation [60]. Refer to Fig. 1.

\subsection{HER-1, HER-3, HER-4}

HER-1 is a non-tissue specific peptide that has been implicated in the oncogenesis of multiple malignancies including breast, colorectal, pancreatic adenocarcinoma, brain glioma multiforme and non-small cell lung cancer [60]. The Food and Drug Administration (FDA) recently approved the use of a novel HER-1 directed tyrosine kinase inhibitor, erlotinab, in conjunction with other established medications for the treatment of advanced pancreatic and non-small cell lung cancers [61]. In addition, cetuximab is an anti-HER-1 monoclonal antibody that has been approved to treat patients with advanced colorectal cancer [62]. Although no anti-tumor vaccines have been formulated that are directed against HER-1, it is a feasible possibility to target this protein in order to develop an anti-HER-1 T cell response. 


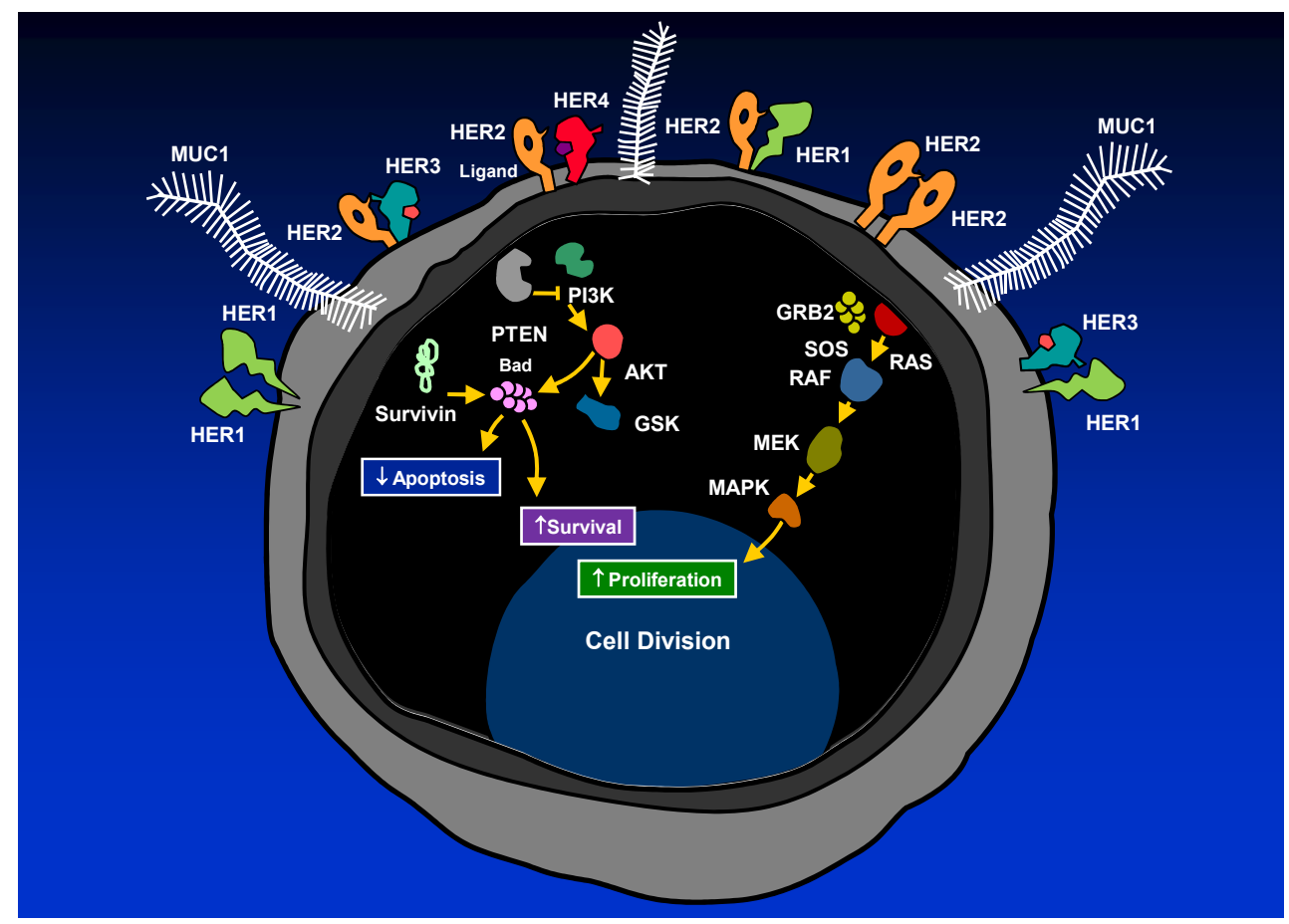

Fig. 1. The EGF receptor family.

HER-3 has a more ambiguous role in tumorigenesis compared to the other members of the EGFR family. It is frequently expressed in breast, ovarian and lung cancers [62-64]. The role of HER-4 in relationship to tumor development is also not clear. HER-4 mutations have recently been shown to augment proliferation and cell survival in melanomas. Agents that target HER-4 may be found to be effective against melanoma and other cancers [60].

\subsection{HER-2}

The HER-2 protein is a well established target of immunotherapy in breast cancer. The proto-oncogene HER-2 is found on chromosome $17 q$ and encodes a transmembrane tyrosine kinase growth factor receptor. HER-2 over expression occurs in ovarian, pancreatic, gastric, lung and head/neck cancers [65-68]. Twenty to thirty percent of breast cancers have been found to amplify the HER-2 gene or overexpress the HER-2 protein, which portends a poorer prognosis and higher risk of recurrence in patients with both invasive and in situ disease [69].

HER-2 represents an ideal target for antigen-specific vaccines used to treat breast cancer. Over expression of this protein is immunogenic as it induces a $\mathrm{T}$ cell immune response causing HER-2 specific antibodies to be present in the serum of breast cancer patient [70]. 
The feasibility of HER-2 targeted vaccines has been demonstrated in animal models [71]. In addition, a number of phase I and II trials using HER-2 based vaccines of all types have been performed in patients with high risk breast cancer. The vaccines in all of these studies were well tolerated and caused minimal toxicity [18].

\section{Development of dendritic cell vaccines for the treatment and prevention of cancer}

The concept of producing a vaccine aimed at specific breast cancer antigens is theoretically straightforward although the details and execution are obviously complex to carry out. There are several different vaccine approaches under investigation for the treatment of early and late stage breast cancer (dendritic cells, whole tumor cells, peptide-based and viralbased) $[72,73]$. Breast cancer vaccines utilizing all of these different strategies are in various stages of development $[18,38,72-74]$. A comprehensive review of the published data on all breast cancer vaccines is beyond the scope of this chapter.

The advent of dendritic cell (DC) vaccines, propelled by the ability to culture human DC cells, has provided promise for a novel vaccine strategies [75]. Despite preclinical evidence and high expectations for the potential effectiveness of DC-based cancer vaccines, initial results of clinical trials were somewhat disappointing with discordant tumor response rates $[43,76,77]$. However, continued interest in therapeutic possibilities of DC vaccines has led to recent successes and promising data in breast and other cancers. The efficacy of DC vaccines continues to improve as efforts have been made to optimize DC vaccines and circumvent tumor escape mechanisms.

\subsection{Dendritic cells}

DC vaccines represent one of a number of strategies for vaccinating patients against tumorassociated antigens. DCs are the most powerful of the APCs and are the primary means by which naïve T cells become immunized to specific antigens. DCs are unique in their ability to activate both the innate and adaptive immune systems. Immature DCs arise from progenitors in the bone marrow and then enter the blood stream and circulate throughout the peripheral tissues where they are exposed to foreign antigens. After capturing antigens, DCs undergo a maturation process that ultimately guides their travel to secondary lymphoid tissues. Once in the regional lymph nodes, the DCs process the captured antigen and then display the antigen as a peptide on their MHC molecules. DCs present the peptides to naïve $\mathrm{T}$ cells resulting in T lymphocyte expansion and differentiation.

In addition to $\mathrm{T}$ cell stimulation, contact with DCs causes activation of B lymphocytes which leads them to differentiate into plasma cells that subsequently release antibodies targeted against the initial pathogen. After antigen exposure, DCs also release cytokines which can activate the cells of the innate immune system, including eosinophils, macrophages, and NK cells. In this way, DCs are capable of activating both the innate and adaptive immunity and are central to the communication between the two immune systems [36, 78-80].

\subsection{Production and adminstration of DC vaccines}

No standard protocol exists for the production of DC vaccines, but there are some general components that are often involved. First, the DCs are collected from the patient via 
leukapheresis and then activated ex vivo with the specific tumor antigen and human granulocyte-macrophage colony-stimulating factor (GM-CSF). The activated DCs are then reintroduced to the patient in order to stimulate a T cell response. We have developed a DC vaccine targeting HER-2 and used to treat patients with DCIS. The production process will be briefly described.

First, peripheral blood monocytes are obtained by leukapheresis followed by elutriation under good clinical practice conditions. Monocytes are then cultured overnight in monocyte-macrophage serum-free medium GM-CSF and interleukin (IL)-4. Immature DCs are next pulsed with HER-2 MHC II binding peptides, extracellular and intracellular domain peptides for 12 hours. In order to potentiate the benefits of signaling infectious nonself at the time of vaccine administration, we then sequentially culture the cells with interferon-gamma (IFN- $\gamma$ ) and bacterial lipopolysaccharcharide (LPS), a TLR agonist, prior to pulsing with MHC class I peptides. As mentioned previously, TLR stimulation leads to cytokine release and DC maturation. This activation strategy assures that the DC are able to robustly secrete IL-12 which maximizes their ability to produce IFN- $\gamma$ and a functional T cell response [55].

The major technical disadvantage to the use of DC vaccines relates to the ex-vivo nature of their generation. A number of obstacles must be dealt with in order to make the process successful and efficient [36, 81]. Most importantly, the production of DC must be made individually for each patient. The process is dependent on access to leukapheresis facilities at the treatment center. Leukapheresis is complex procedure that does not always generate consistent results. The procedure is associated with minimal patient morbidity including, brief electrolyte imbalances, minimal risk of infection and vascular injury. Another important consideration in the production of DC vaccines is the financial burden of manufacturing and administering such an individualized treatment. Sipuleucel-T, the only cancer vaccine that is currently approved by the FDA, is priced at $\$ 31,000$ and is typically given three times, making a complete treatment cost $\$ 93,000$. This is one of the most expensive cancer therapies ever approved $[77,82]$.

We advocate injecting the vaccines directly into distal lymph nodes. It has been shown that only a proportion of peripherally administered DCs migrate to regional lymph nodes [83]. Using ultrasound guidance to inject the cultured DCs directly into distal lymph nodes assures that a predetermined and defined quantity of antigen-loaded DC cells are delivered to the site of T-cell sensitization, thus allowing the vaccine to activate an adaptive immune response. This method also synchronizes peak IL-2 secretion to occur when the DCs are close to T cells. Vaccinating patients prior to surgical resection also allows us to have the opportunity to review the histopathological effects of vaccination on the tumor cells [36].

In April 2010, the FDA approved the first cancer vaccine. This individualized DC vaccine, Sipuleucel-T (Provenge; Dendreon Corp.), is approved for use in men with metastatic castration-resistant prostate cancer. Similar to other DC vaccines, the production of Sipuleucel-T begins with leukaphoresis and the isolation of peripheral blood mononuclear cells. The DCs are then activated by exposure to a recombinant fusion protein (prostatic acid phosphatase and GM-CSF). The treated cells are re-infused into the patient. This process is repeated every two weeks for a total of three treatments [84]. 
The results from initial trials reported a $41 \%$ relative reduction in the risk of death and a trend toward increased survival in the men treated with Sipuleucel-T compared to the men in the placebo group [85, 86]. The IMPACT study, a double-blind, placebo-controlled, multicenter trial involving 512 men with metastatic castration-resistant prostate cancer, was designed with overall survival as the primary endpoint. Of note, these men were either asymptomatic or minimally symptomatic, representing a patient population that is an earlier stage and therefore more likely to be amenable to immunotherapy. Compared with the placebo arm, patients in the Sipuleucel-T arm achieved a $22 \%$ relative reduction in the risk of death. This represented a 4.1 month increase in median overall survival between the placebo and Sipuleucel-T arms. There was no significant difference achieved in the prostatespecific antigen response or time to progression between the two arms. Sipuleucel-T was well tolerated [84].

\section{DC vaccination in breast cancer}

Successful reduction of HER-2 over expressing tumors requires activation of both the innate and adaptive immune responses [87]. As described, DCs are unique in their ability to elicit responses from tumor-specific effector and memory T cells. Numerous strategies exist that aim to introduce tumor antigens into DCs in order to generate vaccines (loading individual tumor peptides, transfer of tumor-specific DNA or RNA through lipofection or viral vectors, whole tumor cell fusion). An early DC vaccine strategy for breast cancer involved the production of DC/tumor cell fusion vaccines. Avigen et al conducted a trial in which 16 patients with metastatic breast cancer were injected with a fusion vaccine using tumor cells obtained from a biopsy and DCs acquired from leukapheresis [88]. Three patients had a significant clinical response. Unfortunately the efficacy of fusion cell generation is less than $45 \%$ and multiple patients were not able to receive the full course of vaccines due to limited yield.

In 2007, Park et al published data from a phase I study in which they treated 18 metastatic breast cancer patients with Lapuleucel-T (Dendreon Corp), a vaccine produced in a similar fashion as sipuleucel-T, although a HER-2 fusion protein is used [89]. The study was designed to evaluate the safety and immunologic activity of the novel agent. The therapy was well tolerated. Significant immune responses were stimulated (as measured by lymphocyte proliferation and IFN- $\gamma$ enzyme-linked immunospot assays). The therapy was also associated with tumor response and extended disease stabilization. Further trials are warranted to determine the efficacy in patients with earlier stage disease and in combination with other anti-HER-2 therapies.

Another DC vaccine approach that is being explored is Adevexin (Introgen Therapuetics), made form leukapheresed APCs that are transfected with a replication-impaired adenoviral vector that brings the p53 gene under control of a cytomegalovirus promoter [90]. This vaccine is not specifically anti-HER-2, but instead works under the theory that p53 restoration can be used to treat cancer. This vaccine triggers p53-specific T-cell responses against cancer cells with mutant p53 and has proven to be safe and synergistically effective in a number of tumor types.

Currently other anti-HER-2 DC vaccines trials are recruiting patients and results of these trials are pending [74]. We are conducting a clinical trial with a novel DC vaccine designed 
to treat DCIS patients with lesions that overexpress HER-2. No other DC-based vaccines have been designed specifically to treat HER-2 expressing DCIS tumors. We anticipate a significant reduction in disease burden in our patients after a complete vaccine course. We hope that this vaccine will also be preventative in terms of both disease recurrence and rate of transformation into invasive breast cancer.

Our strategy of vaccine production utilizes both MHC I and II peptides as well as ex vivo activation with IFN- $\gamma$ and LPS to yield polarized DC cells that induce a unique set of soluble factors including high levels of IL-12 and Th1 chemokines not elicited through traditional vaccines methods. This innovative DC vaccine strategy called Immune Conditioning by Activated Innate Transfer (ICAIT) uses monocyte-derived DCs that are activated with and a special clinical-grade TLR 4 ligand, LPS and IFN-ץ (ICAIT-DC). This unique DC activation method gives the DCs qualities that are not found in the so-called "gold-standard" DCs used in prior vaccine trials. The "gold standard" DC vaccines, activated with TNF, IL-6, PGE2, IL-1 $\beta$, have the potential to simulate aseptic inflammation [91]. In contrast, ICAITDCs produce high levels of factors that specifically enhance aspects of anti-tumor immunity such as NK cells which augment tumor rejection, and TNF and IL-12 which are antiangiogenic [55, 92]. ICAIT-DCs also have the distinct ability to influence the quality of sensitized $\mathrm{T}$ cells and can condition $\mathrm{T}$ cells for recognition of HER-2 expressing tumors. Lastly, ICAIT-DCs posses a killer function that enables them to lyse breast cancer cells.

Our DC vaccine is unique in its design against DCIS rather than invasive breast cancer. Our first neoadjuvant trial involved treating patients with HER-2 expressing DCIS tumors. The patients were treated with a course of four weekly intranodal injections of ICAIT-DCs that had been pulsed with HER-2 derived proteins. This approach yielded promising results that have positive implications for the treatment and prevention of high risk breast cancers. Specifically, 85\% of ICAIT-treated patients developed immune responses to at least one of the HER-2 peptides. Eleven of the 22 patients with residual DCIS treated with the vaccine in our initial studies showed loss of HER-2 expression and tumor regression. The immunized patients developed a specific immune response against the HER-derived peptides and presented high levels of CD4+ and CD8+ $\mathrm{T}$ lymphocytes. These results have potential positive implications not only for prognosis but also in terms of breast-conserving surgery [55]. Five of the 27 patients had no evidence of remaining disease. The vaccination was most effective in patients with hormone-independent DCIS as $40 \%$ of ER negative HER-2 positive patients had no residual disease whereas only $5 \%$ of ER positive HER-2 positive had no residual disease. The vaccine appeared to alter the phenotype of the remaining DCIS in the patients that were found to have residual disease. The rate of change to a different postvaccination phenotype was significantly different between the ER positive and ER negative patients. $43.8 \%$ of the patients that were initially ER positive and HER-2 positive phenotype converted to ER positive and HER-2 negative phenotype. In comparison, $50 \%$ of the patients that initially had tumors that were ER negative and HER-2 positive changed to the ER negative and HER-2 negative phenotype. These results supported the safety and efficacy of the DC based HER-2 vaccine. The vaccine induced a decline or eradication of HER-2 expression (work not yet published).

\section{Future direction and prevention}

There are a number of molecules that have been discovered to be present in breast carcinomas that have not yet been exploited to their fullest potential. The future directions 
for the development of breast cancer vaccines should focus not only on targeting molecules that are specific tumor related antigens, but also to the downstream signaling pathways involved in tumorigenesis. For example, recent work has elucidated the role of survivin (Figure 1), a protein in the anti-apoptotic family. Blocking the expression of survivin, was found to have a direct role in the initiation of apoptosis of breast cancer cells [93]. Upregulation of survivin is also directly linked to HER-2 over expression [94]. A novel antisurvivin based therapy in combination with an anti-HER-2 DC vaccine is an exciting possibility to treat and possibly prevent breast cancers.

Another promising possibility is to develop immune responses against other HER family members including HER-1 and HER-3. HER-3 has no ligand binding sites but intracellular signaling moieties and can partner with HER-2 and HER-1. HER-3 signaling may lead to HER-2 resistance thus developing vaccines against these targets may supplement HER-2 vaccines to make them better preventive agents eliminating vaccine escapes.

MUC-1(Figure 1), an epithelial glycoprotein that is over expressed in may breast cancers, is another molecule that has yet to be fully exploited as an anti-cancer drug target. This molecule has been implicated in tumor invasion and metastases [95]. MUC-1 pulsed DC based vaccine trials for patients with pancreatic and biliary carcinomas are currently underway and the clinical efficacy of these vaccines is not known at this time [96]. MUC-1 is another molecule that could potentially used to target breast cancers.

There is immeasurable potential for vaccine therapy to be used in immunocompetent patients with minimal disease to prevent disease progression and recurrence. Even more exciting is the potential to treat patients with no disease at all. Forty percent of the participants in our recent vaccine trial converted from ER positive HER-2 positive to ER positve HER-2 negative suggesting that HER-2 vaccines can direct or steer tumors to more favorable phenotypes. The ultimate goal is therefore to produce a vaccine that could prevent breast cancer formation altogether. The development of successful breast cancer prevention would be applicable to other solid tumor malignancies such as colorectal, head and neck cancer, lung cancer, gastric cancer and other GI malignancies.

\section{References}

[1] Nathanson, N. and O.M. Kew, From emergence to eradication: the epidemiology of poliomyelitis deconstructed. Am J Epidemiol. 172(11): p. 1213-29.

[2] Schiller, J.T. and D.R. Lowy, Vaccines to prevent infections by oncoviruses. Annu Rev Microbiol. 64: p. 23-41.

[3] Jemal, A., et al., Global cancer statistics. CA Cancer J Clin. 61(2): p. 69-90.

[4] Dawood, S., et al., Defining breast cancer prognosis based on molecular phenotypes: results from a large cohort study. Breast Cancer Res Treat. 126(1): p. 185-92.

[5] Sorlie, T., et al., Gene expression patterns of breast carcinomas distinguish tumor subclasses with clinical implications. Proc Natl Acad Sci U S A, 2001. 98(19): p. 10869-74.

[6] Sorlie, T., et al., Repeated observation of breast tumor subtypes in independent gene expression data sets. Proc Natl Acad Sci U S A, 2003. 100(14): p. 8418-23.

[7] van 't Veer, L.J., et al., Gene expression profiling predicts clinical outcome of breast cancer. Nature, 2002. 415(6871): p. 530-6. 
[8] Perou, C.M., et al., Molecular portraits of human breast tumours. Nature, 2000. 406(6797): p. 747-52.

[9] Sotiriou, C. and L. Pusztai, Gene-expression signatures in breast cancer. N Engl J Med, 2009. 360(8): p. 790-800.

[10] Holmes, P., et al., Prognostic markers and long-term outcomes in ductal carcinoma in situ of the breast treated with excision alone. Cancer.

[11] Roses, R.E., et al., HER-2/neu overexpression as a predictor for the transition from in situ to invasive breast cancer. Cancer Epidemiol Biomarkers Prev, 2009. 18(5): p. 1386-9.

[12] Panet-Raymond, V., et al., Clinicopathologic factors of the recurrent tumor predict outcome in patients with ipsilateral breast tumor recurrence. Cancer. 117(10): p. 2035-43.

[13] Kerlikowske, K., et al., Biomarker expression and risk of subsequent tumors after initial ductal carcinoma in situ diagnosis. J Natl Cancer Inst. 102(9): p. 627-37.

[14] Carlson, R.W., et al., Invasive breast cancer. J Natl Compr Canc Netw. 9(2): p. 136-222.

[15] Lee, S.C., et al., Natural killer (NK):dendritic cell (DC) cross talk induced by therapeutic monoclonal antibody triggers tumor antigen-specific $T$ cell immunity. Immunol Res. 50(2-3): p. 248-54.

[16] Madarnas, Y., et al., Adjuvant/neoadjuvant trastuzumab therapy in women with HER-2/neuoverexpressing breast cancer: a systematic review. Cancer Treat Rev, 2008. 34(6): p. 53957.

[17] Haffty, B.G., et al., Locoregional relapse and distant metastasis in conservatively managed triple negative early-stage breast cancer. J Clin Oncol, 2006. 24(36): p. 5652-7.

[18] Anderson, K.S., Tumor vaccines for breast cancer. Cancer Invest, 2009. 27(4): p. 361-8.

[19] DeNardo, D.G. and L.M. Coussens, Inflammation and breast cancer. Balancing immune response: crosstalk between adaptive and innate immune cells during breast cancer progression. Breast Cancer Res, 2007. 9(4): p. 212.

[20] de Visser, K.E. and L.M. Coussens, The interplay between innate and adaptive immunity regulates cancer development. Cancer Immunol Immunother, 2005. 54(11): p. 1143-52.

[21] Coussens, L.M. and Z. Werb, Inflammation and cancer. Nature, 2002. 420(6917): p. 860-7.

[22] Johansson, M., et al., Immune cells as anti-cancer therapeutic targets and tools. J Cell Biochem, 2007. 101(4): p. 918-26.

[23] Disis, M.L. and K.H. Park, Immunomodulation of breast cancer via tumor antigen specific Th1. Cancer Res Treat, 2009. 41(3): p. 117-21.

[24] Almand, B., et al., Increased production of immature myeloid cells in cancer patients: a mechanism of immunosuppression in cancer. J Immunol, 2001. 166(1): p. 678-89.

[25] Serafini, P., et al., Derangement of immune responses by myeloid suppressor cells. Cancer Immunol Immunother, 2004. 53(2): p. 64-72.

[26] Gabrilovich, D.I., et al., Mechanism of immune dysfunction in cancer mediated by immature Gr-1+ myeloid cells. J Immunol, 2001. 166(9): p. 5398-406.

[27] Yang, L., et al., Expansion of myeloid immune suppressor Gr+CD11b+ cells in tumor-bearing host directly promotes tumor angiogenesis. Cancer Cell, 2004. 6(4): p. 409-21.

[28] Ernst, P.B. and B.D. Gold, The disease spectrum of Helicobacter pylori: the immunopathogenesis of gastroduodenal ulcer and gastric cancer. Annu Rev Microbiol, 2000. 54: p. 615-40.

[29] Kuper, H., H.O. Adami, and D. Trichopoulos, Infections as a major preventable cause of human cancer. J Intern Med, 2000. 248(3): p. 171-83. 
[30] Shacter, E. and S.A. Weitzman, Chronic inflammation and cancer. Oncology (Williston Park), 2002. 16(2): p. 217-26, 229; discussion 230-2.

[31] Peek, R.M., Jr., S. Mohla, and R.N. DuBois, Inflammation in the genesis and perpetuation of cancer: summary and recommendations from a national cancer institute-sponsored meeting. Cancer Res, 2005. 65(19): p. 8583-6.

[32] Tlsty, T.D. and L.M. Coussens, Tumor stroma and regulation of cancer development. Annu Rev Pathol, 2006. 1: p. 119-50.

[33] Zou, W., Immunosuppressive networks in the tumour environment and their therapeutic relevance. Nat Rev Cancer, 2005. 5(4): p. 263-74.

[34] Dunn, G.P., L.J. Old, and R.D. Schreiber, The three Es of cancer immunoediting. Annu Rev Immunol, 2004. 22: p. 329-60.

[35] Dunn, G.P., et al., Cancer immunoediting: from immunosurveillance to tumor escape. Nat Immunol, 2002. 3(11): p. 991-8.

[36] Koski, G.K., et al., Reengineering dendritic cell-based anti-cancer vaccines. Immunol Rev, 2008. 222: p. 256-76.

[37] Boon, T., et al., Tumor antigens recognized by T lymphocytes. Annu Rev Immunol, 1994. 12: p. 337-65.

[38] Mittendorf, E.A., G.E. Peoples, and S.E. Singletary, Breast cancer vaccines: promise for the future or pipe dream? Cancer, 2007. 110(8): p. 1677-86.

[39] Disis, M.L., et al., Existent T-cell and antibody immunity to HER-2/neu protein in patients with breast cancer. Cancer Res, 1994. 54(1): p. 16-20.

[40] Jerome, K.R., N. Domenech, and O.J. Finn, Tumor-specific cytotoxic T cell clones from patients with breast and pancreatic adenocarcinoma recognize EBV-immortalized B cells transfected with polymorphic epithelial mucin complementary DNA. J Immunol, 1993. 151(3): p. 1654-62.

[41] Menard, S., et al., Lymphoid infiltration as a prognostic variable for early-onset breast carcinomas. Clin Cancer Res, 1997. 3(5): p. 817-9.

[42] Mahmoud, S.M., et al., Tumor-Infiltrating CD8+ Lymphocytes Predict Clinical Outcome in Breast Cancer. J Clin Oncol. 29(15): p. 1949-55.

[43] Draube, A., et al., Dendritic cell based tumor vaccination in prostate and renal cell cancer: $a$ systematic review and meta-analysis. PLoS One. 6(4): p. e18801.

[44] Disis, M.L., et al., Effect of dose on immune response in patients vaccinated with an her-2/neu intracellular domain protein--based vaccine. J Clin Oncol, 2004. 22(10): p. 1916-25.

[45] Hakim, F.T., et al., Constraints on CD4 recovery postchemotherapy in adults: thymic insufficiency and apoptotic decline of expanded peripheral CD4 cells. Blood, 1997. 90(9): p. 3789-98.

[46] Mellios, T., H.L. Ko, and J. Beuth, Impact of adjuvant chemo- and radiotherapy on the cellular immune system of breast cancer patients. In Vivo. 24(2): p. 227-30.

[47] Rotstein, S., et al., Long term effects on the immune system following local radiation therapy for breast cancer. I. Cellular composition of the peripheral blood lymphocyte population. Int J Radiat Oncol Biol Phys, 1985. 11(5): p. 921-5.

[48] Czerniecki, B.J., R.E. Roses, and G.K. Koski, Development of vaccines for high-risk ductal carcinoma in situ of the breast. Cancer Res, 2007. 67(14): p. 6531-4.

[49] Altintas, S., et al., Prognostic significance of oncogenic markers in ductal carcinoma in situ of the breast: a clinicopathologic study. Breast J, 2009. 15(2): p. 120-32. 
[50] Tamimi, R.M., et al., Comparison of molecular phenotypes of ductal carcinoma in situ and invasive breast cancer. Breast Cancer Res, 2008. 10(4): p. R67.

[51] Zafrani, B., et al., Mammographically-detected ductal in situ carcinoma of the breast analyzed with a new classification. A study of 127 cases: correlation with estrogen and progesterone receptors, p53 and c-erbB-2 proteins, and proliferative activity. Semin Diagn Pathol, 1994. 11(3): p. 208-14.

[52] Evans, A.J., et al., Correlations between the mammographic features of ductal carcinoma in situ (DCIS) and C-erbB-2 oncogene expression. Nottingham Breast Team. Clin Radiol, 1994. 49(8): p. 559-62.

[53] Clark, S.E., et al., Molecular subtyping of DCIS: heterogeneity of breast cancer reflected in pre-invasive disease. Br J Cancer. 104(1): p. 120-7.

[54] Dawson, S.J., et al., BCL2 in breast cancer: a favourable prognostic marker across molecular subtypes and independent of adjuvant therapy received. Br J Cancer. 103(5): p. 668-75.

[55] Czerniecki, B.J., et al., Targeting HER-2/neu in early breast cancer development using dendritic cells with staged interleukin-12 burst secretion. Cancer Res, 2007. 67(4): p. 1842-52.

[56] Otto, K., et al., Lack of toxicity of therapy-induced $T$ cell responses against the universal tumour antigen survivin. Vaccine, 2005. 23(7): p. 884-9.

[57] Morse, M.A., et al., Immunotherapy with autologous, human dendritic cells transfected with carcinoembryonic antigen mRNA. Cancer Invest, 2003. 21(3): p. 341-9.

[58] Brossart, P., et al., Induction of cytotoxic T-lymphocyte responses in vivo after vaccinations with peptide-pulsed dendritic cells. Blood, 2000. 96(9): p. 3102-8.

[59] Hynes, N.E. and G. MacDonald, ErbB receptors and signaling pathways in cancer. Curr Opin Cell Biol, 2009. 21(2): p. 177-84.

[60] Lee, M.K.t., A. Sharma, and B.J. Czerniecki, It's all in for the HER family in tumorigenesis. Expert Rev Vaccines. 9(1): p. 29-34.

[61] Mendelsohn, J. and J. Baselga, Epidermal growth factor receptor targeting in cancer. Semin Oncol, 2006. 33(4): p. 369-85.

[62] Harding, J. and B. Burtness, Cetuximab: an epidermal growth factor receptor chemeric human-murine monoclonal antibody. Drugs Today (Barc), 2005. 41(2): p. 107-27.

[63] Tanner, B., et al., ErbB-3 predicts survival in ovarian cancer. J Clin Oncol, 2006. 24(26): p. 4317-23.

[64] Engelman, J.A., et al., ErbB-3 mediates phosphoinositide 3-kinase activity in gefitinibsensitive non-small cell lung cancer cell lines. Proc Natl Acad Sci U S A, 2005. 102(10): p. 3788-93.

[65] Slamon, D.J., et al., Studies of the HER-2/neu proto-oncogene in human breast and ovarian cancer. Science, 1989. 244(4905): p. 707-12.

[66] Williams, T.M., et al., Expression of c-erbB-2 in human pancreatic adenocarcinomas. Pathobiology, 1991. 59(1): p. 46-52.

[67] Holbro, T., G. Civenni, and N.E. Hynes, The ErbB receptors and their role in cancer progression. Exp Cell Res, 2003. 284(1): p. 99-110.

[68] Ibrahim, S.O., et al., Expression of c-erbB proto-oncogene family members in squamous cell carcinoma of the head and neck. Anticancer Res, 1997. 17(6D): p. 4539-46.

[69] Ross, J.S. and J.A. Fletcher, The HER-2/neu Oncogene in Breast Cancer: Prognostic Factor, Predictive Factor, and Target for Therapy. Oncologist, 1998. 3(4): p. 237-252. 
[70] Disis, M.L., et al., High-titer HER-2/neu protein-specific antibody can be detected in patients with early-stage breast cancer. J Clin Oncol, 1997. 15(11): p. 3363-7.

[71] Park, J.M., et al., Early role of CD4+ Th1 cells and antibodies in HER-2 adenovirus vaccine protection against autochthonous mammary carcinomas. J Immunol, 2005. 174(7): p. 4228-36.

[72] Shumway, N.M., et al., Therapeutic breast cancer vaccines: a new strategy for early-stage disease. BioDrugs, 2009. 23(5): p. 277-87.

[73] Soliman, H., Developing an effective breast cancer vaccine. Cancer Control. 17(3): p. 183-90.

[74] Ladjemi, M.Z., et al., Anti-HER2 vaccines: new prospects for breast cancer therapy. Cancer Immunol Immunother. 59(9): p. 1295-312.

[75] Romani, N., et al., Proliferating dendritic cell progenitors in human blood. J Exp Med, 1994. 180(1): p. 83-93.

[76] Palucka, K., H. Ueno, and J. Banchereau, Recent developments in cancer vaccines. J Immunol. 186(3): p. 1325-31.

[77] Gulley, J.L. and C.G. Drake, Immunotherapy for prostate cancer: recent advances, lessons learned, and areas for further research. Clin Cancer Res. 17(12): p. 3884-91.

[78] Dallal, R.M. and M.T. Lotze, The dendritic cell and human cancer vaccines. Curr Opin Immunol, 2000. 12(5): p. 583-8.

[79] Palucka, K. and J. Banchereau, Dendritic cells: a link between innate and adaptive immunity. J Clin Immunol, 1999. 19(1): p. 12-25.

[80] Palucka, K., et al., Dendritic cells and immunity against cancer. J Intern Med. 269(1): p. 6473.

[81] Andrews, D.M., E. Maraskovsky, and M.J. Smyth, Cancer vaccines for established cancer: how to make them better? Immunol Rev, 2008. 222: p. 242-55.

[82] Chambers, J.D. and P.J. Neumann, Listening to Provenge--what a costly cancer treatment says about future Medicare policy. N Engl J Med. 364(18): p. 1687-9.

[83] De Vries, I.J., et al., Effective migration of antigen-pulsed dendritic cells to lymph nodes in melanoma patients is determined by their maturation state. Cancer Res, 2003. 63(1): p. 12-7.

[84] Kantoff, P.W., et al., Sipuleucel-T immunotherapy for castration-resistant prostate cancer. N Engl J Med. 363(5): p. 411-22.

[85] Higano, C.S., et al., Integrated data from 2 randomized, double-blind, placebo-controlled, phase 3 trials of active cellular immunotherapy with sipuleucel-T in advanced prostate cancer. Cancer, 2009. 115(16): p. 3670-9.

[86] Small, E.J., et al., Placebo-controlled phase III trial of immunologic therapy with sipuleucel-T (APC8015) in patients with metastatic, asymptomatic hormone refractory prostate cancer. $\mathrm{J}$ Clin Oncol, 2006. 24(19): p. 3089-94.

[87] Wiedermann, U., et al., A virosomal formulated Her-2/neu multi-peptide vaccine induces Her-2/neu-specific immune responses in patients with metastatic breast cancer: a phase I study. Breast Cancer Res Treat. 119(3): p. 673-83.

[88] Avigan, D., et al., Fusion cell vaccination of patients with metastatic breast and renal cancer induces immunological and clinical responses. Clin Cancer Res, 2004. 10(14): p. 4699708.

[89] Park, J.W., et al., Treatment with autologous antigen-presenting cells activated with the HER2 based antigen Lapuleucel-T: results of a phase I study in immunologic and clinical activity in HER-2 overexpressing breast cancer. J Clin Oncol, 2007. 25(24): p. 3680-7. 
[90] Senzer, N. and J. Nemunaitis, A review of contusugene ladenovec (Advexin) p53 therapy. Curr Opin Mol Ther, 2009. 11(1): p. 54-61.

[91] Lombardi, V., et al., Human dendritic cells stimulated via TLR7 and/or TLR8 induce the sequential production of Il-10, IFN-gamma, and IL-17A by naive CD4+ T cells. J Immunol, 2009. 182(6): p. 3372-9.

[92] Albini, A., et al., Angiostatin anti-angiogenesis requires IL-12: the innate immune system as a key target. J Transl Med, 2009. 7: p. 5.

[93] Gritsko, T., et al., Persistent activation of stat3 signaling induces survivin gene expression and confers resistance to apoptosis in human breast cancer cells. Clin Cancer Res, 2006. 12(1): p. 11-9.

[94] Siddiqa, A., et al., Expression of HER-2 in MCF-7 breast cancer cells modulates antiapoptotic proteins Survivin and Bcl-2 via the extracellular signal-related kinase (ERK) and phosphoinositide-3 kinase (PI3K) signalling pathways. BMC Cancer, 2008. 8: p. 129.

[95] Wierecky, J., M. Mueller, and P. Brossart, Dendritic cell-based cancer immunotherapy targeting MUC-1. Cancer Immunol Immunother, 2006. 55(1): p. 63-7.

[96] Lepisto, A.J., et al., A phase I/II study of a MUC1 peptide pulsed autologous dendritic cell vaccine as adjuvant therapy in patients with resected pancreatic and biliary tumors. Cancer Ther, 2008. 6(B): p. 955-964. 


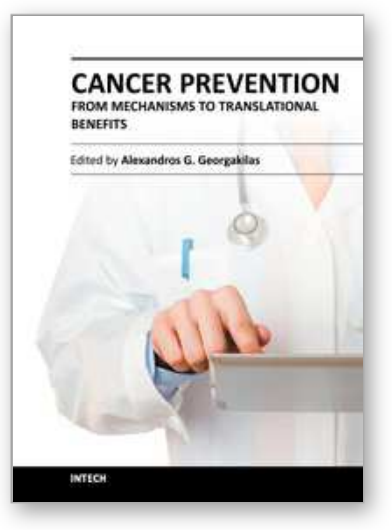

\author{
Cancer Prevention - From Mechanisms to Translational Benefits \\ Edited by Dr. Alexandros G. Georgakilas
}

ISBN 978-953-51-0547-3

Hard cover, 476 pages

Publisher InTech

Published online 20, April, 2012

Published in print edition April, 2012

This unique synthesis of chapters from top experts in their fields targets the unique and significant area of cancer prevention for different types of cancers. Perspective readers are invited to go through novel ideas and current developments in the field of molecular mechanisms for cancer prevention, epidemiological studies, antioxidant therapies and diets, as well as clinical aspects and new advances in prognosis and avoidance of cancer. The primary target audience for the book includes PhD students, researchers, biologists, medical doctors and professionals who are interested in mechanistic studies on cancer prevention and translational benefits for optimized cancer treatment.

\title{
How to reference
}

In order to correctly reference this scholarly work, feel free to copy and paste the following:

Shayna Showalter and Brian J. Czerniecki (2012). Targeting Molecular Pathways for Prevention of High Risk Breast Cancer: A Model for Cancer Prevention, Cancer Prevention - From Mechanisms to Translational Benefits, Dr. Alexandros G. Georgakilas (Ed.), ISBN: 978-953-51-0547-3, InTech, Available from: http://www.intechopen.com/books/cancer-prevention-from-mechanisms-to-translational-benefits/dendriticcells-vaccines-for-prevention-of-estrogen-independent-breast-cancer-a-model-for-cancer-pre

\section{INTECH}

open science | open minds

\section{InTech Europe}

University Campus STeP Ri

Slavka Krautzeka 83/A

51000 Rijeka, Croatia

Phone: +385 (51) 770447

Fax: +385 (51) 686166

www.intechopen.com

\section{InTech China}

Unit 405, Office Block, Hotel Equatorial Shanghai

No.65, Yan An Road (West), Shanghai, 200040, China

中国上海市延安西路65号上海国际贵都大饭店办公楼 405 单元

Phone: +86-21-62489820

Fax: +86-21-62489821 
(C) 2012 The Author(s). Licensee IntechOpen. This is an open access article distributed under the terms of the Creative Commons Attribution 3.0 License, which permits unrestricted use, distribution, and reproduction in any medium, provided the original work is properly cited. 